\title{
Registerempfehlungen - damit Daten von Nutzen sind
}

\author{
Christoph Bosshard \\ Dr. med., Vizepräsident der FMH, Departementsverantwortlicher Daten, Demographie und Qualität DDQ
}

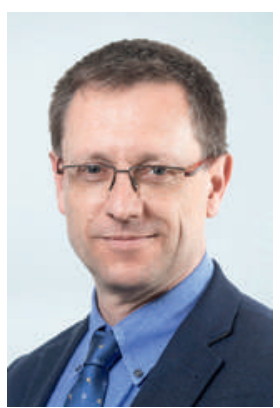

«Manchmal ist es zum Verzweifeln», höre ich immer wieder von Kolleginnen und Kollegen, welche unter der zunehmenden Flut von Administration leiden und mit ihnen auch die Patienten, welche dabei zu kurz kommen. «Und jetzt kommst du mit noch mehr Dokumentationsaufwand», wird auch begreiflicherweise nachgeschoben, wenn ich mit einer neuen, aus meiner Sicht ansprechenden Idee punkto Register vorstellig werde. Spätestens dann realisiere ich, wo die praktischen Grenzen liegen: Um sowohl Ärzte wie auch Patienten für solchen Mehraufwand zu überzeugen, muss nicht nur dieser minimiert sein, sondern es muss auch ein klarer Mehrwert für die Beteiligten nachweisbar sein. Zudem

Die erhobenen Daten müssen nachweislich nützlich sein. Zugleich hat ihre Interpretation und Verwendung mit Umsicht zu geschehen.

ist eine Vertrauensbasis zu schaffen, dass die Daten nicht nur in vereinbarter Form bearbeitet werden, sondern auch deren Interpretation und Verwendung mit der notwendigen Umsicht geschehen. Denn wir alle wissen: Jede Erkenntnis hat ihre Grenzen. Diese zu kennen ist genauso wichtig wie die Botschaft selbst. Wie beispielsweise in der Overuse-Thematik gibt es auch diverse andere Forschungsgebiete, wo Aussagen lediglich retrospektiv möglich sind. Dies ist wissenschaftliche Realität. Wir alle leben unser Leben jedoch vorwärts. Das Wissen, woher wir kommen, soll uns das Finden unseres Kurses für die Zukunft erleichtern. Gehen müssen wir diesen Weg jedoch selbst. Hier ist auch stets unsere Erfahrung, unser ärztliches Handeln unter sorgfältiger Abwägung zusammen mit unseren Patientinnen und Patienten gefragt.

Am 29. August 2012 publizierte die FMH ein Grundlagenpapier zur Thematik Register, welches mitgeholfen hat, Überlegungen zu dieser Thematik in Bewegung zu bringen. Um breit abgestützt Konsens punkto der diversen Standards zu Registern zu finden, hat sich eine Arbeitsgruppe bestehend aus den fünf Herausgeberorganisationen ANQ, H+, SAMW und unimedsuisse und FMH auf den Weg gemacht und schliesslich unter breitester Vernehmlassung und Einarbeitung von ca. 160 Rück- meldungen Empfehlungen zum Aufbau und Betrieb von gesundheitsbezogenen Registern verabschiedet. Dieser Bottom-up-Prozess soll Verwaltung und Politik aufzeigen, was benötigt wird, um gute Aussagen zu ermöglichen. Letztlich sind auch hier die Ressourcen ein wichtiges Thema.

Damit gesundheitsbezogene Register effektiv auf ihre Qualität überprüfbar sind, haben die Herausgeber minimale Standards erarbeitet. Diese zielen darauf ab, dass die Erfassung und Verwendung der Daten nach klar definierten Kriterien erfolgen und die Datenqualität gesichert ist. Weiter werden die Kompetenzen zur Führung solcher Register thematisiert. Die Empfehlungen sollen auch dazu beitragen, dass die Persönlichkeitsrechte der datenspendenden Personen jederzeit gewahrt bleiben Schliesslich ermöglicht die Anwendung der Minimalstandards eine effiziente Datenerhebung und steigert deren Nutzen. So können Doppelspurigkeiten verringert, Synergien zwischen Datenerhebungsstellen besser genutzt und die Qualität und Aussagekraft der Daten erhöht werden.

Dass die Ärzteschaft hier bereits Tatbeweise erbracht hat, belegen diverse Aktivitäten, welche durch die ärztlichen Organisationen oder individuell angestossen oder mitgetragen werden. Eine Übersicht über die ak-

\section{Damit gesundheitsbezogene Register auf} ihre Qualität überprüfbar sind, empfehlen sich minimale Standards.

tuelle Situation bietet das Forum medizinische Register der FMH unter www.saqm.ch $\rightarrow$ Themenfelder $\rightarrow$ Wissenstransfer. Mit den in der Schweizerischen Ärztezeitung vom 12. Oktober 2016 publizierten gemeinsamen Empfehlungen zu den gesundheitsbezogenen Registern will die FMH mithelfen, ein weiteres Mosaiksteinchen ins bunte Bild der Qualitätslandschaft einzusetzen, wie ihre Mitglieder und sie dies von jeher und täglich immer wieder tun. Sie finden die gemeinsamen Empfehlungen von $\mathrm{H}+$, ANQ, SAMW, unimedsuisse und FMH auf www.saqm.ch $\rightarrow$ Publikationen $\rightarrow$ Empfehlungen Register. 